\title{
The functional equation for $L$-functions of hyperelliptic curves
}

\author{
Michel Börner, Irene I. Bouw, and Stefan Wewers
}

\begin{abstract}
We compute the $L$-functions of a large class of algebraic curves, and verify the expected functional equation numerically. Our computations are based on our previous results on stable reduction to calculate the local $L$-factor and the conductor exponent at the primes of bad reduction. Most of our examples are hyperelliptic curves of genus $g \geq 2$ defined over $\mathbb{Q}$ which have semistable reduction at every prime $p$. We also treat a few more general examples of superelliptic curves.
\end{abstract}

2010 Mathematics Subject Classification. Primary 11G40. Secondary: 14G10, 11G20.

\section{Introduction}

1.1 Let $Y$ be a smooth projective curve of genus $g \geq 2$ over a number field $K$. The $L$-function of $Y$ is an analytic function of one complex variable $s$ defined for $\Re(s) \geq 2$ as an Euler product

$$
L(Y, s):=\prod_{\mathfrak{p}} L_{\mathfrak{p}}(Y, s),
$$

where $\mathfrak{p}$ ranges over the prime ideals of $K$ and the local $L$-factor is of the form

$$
L_{\mathfrak{p}}(Y, s)=\frac{1}{P\left((\mathrm{~Np})^{-s}\right)} .
$$

Here $\mathrm{Np}$ is the norm of $\mathfrak{p}$ and $P(T)=1+\ldots \in \mathbb{Z}[T]$ is a polynomial with integer coefficients depending on $\mathfrak{p}$. Another invariant associated with $Y$ and relevant for our discussion is the conductor of the $L$-function. It is a positive real number of the form

$$
N:=\delta_{K}^{2 g} \cdot \prod_{\mathfrak{p}}(\mathrm{Np})^{f_{\mathfrak{p}}},
$$

where $\delta_{K}$ is the discriminant of $K$ and $f_{\mathfrak{p}} \geq 0$ is a nonnegative integer called the conductor exponent at $\mathfrak{p}$, which is zero for almost all $\mathfrak{p}$. We refer to [6], $\S 2$, for precise definitions of $L_{\mathfrak{p}}(Y, s)$ and $f_{\mathfrak{p}}$. 
It is conjectured that $L(Y, s)$ has an analytic continuation to the whole complex plane, and a functional equation of the form

$$
\Lambda(Y, s)= \pm \Lambda(Y, 2-s),
$$

where

$$
\Lambda(Y, s):=N^{s / 2}(2 \pi)^{-g s} \Gamma(s)^{g} L(Y, s) .
$$

1.2 The main motivation for this paper - which continues a project begun in [6] - is the question how to compute the local $L$-factor $L_{\mathfrak{p}}(Y, s)$ and the conductor exponent $f_{\mathfrak{p}}$ explicitly, given the curve $Y$ and the prime $\mathfrak{p}$. If the curve $Y$ has good reduction at $\mathfrak{p}$ (which is true for almost all $\mathfrak{p}$ ) it is well known how to do that. Namely, $f_{\mathfrak{p}}=0$ and $L_{\mathfrak{p}}(Y, s)=P\left(\bar{Y},(\mathrm{~Np})^{-s}\right)^{-1}$, where $P(\bar{Y}, T) \in \mathbb{Z}[T]$ is the numerator of the zeta function of the reduction $\bar{Y}$ of $Y$ at $\mathfrak{p}$. To compute $P(\bar{Y}, T)$ for small primes $\mathfrak{p}$, one can simply count the number of

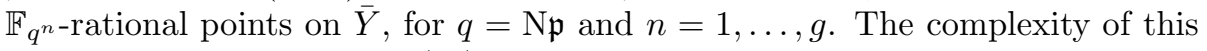
approach is bounded by $\mathcal{O}\left(q^{g}\right)$. There is an extensive literature dealing with various methods for lowering this asymptotic bound, see e.g. [10, 11] or [12.

If $Y$ has bad reduction at $\mathfrak{p}$ then it is not so easy to compute $L_{\mathfrak{p}}(Y, s)$ and $f_{\mathfrak{p}}$ directly from the curve $Y$, even if $\mathrm{Np}$ is very small (say, equal to $p=2$ ). Prior to [6], no general and systematic approach was known except for $g=1$, and for $g=2$ and $q$ odd. Nevertheless, there have been successful attempts to compute $L_{\mathfrak{p}}(Y, s)$ and $f_{\mathfrak{p}}$ for all $\mathfrak{p}$ for curves of genus $g \geq 2$, for instance by Dokchitser, de Jeu and Zagier ([9]), and by Booker ([4, [5]). Without going into details, their method is either based on guessing $L_{\mathfrak{p}}(Y, s)$ and $f_{\mathfrak{p}}$ for the finitely many primes of bad reduction and then verifying this guess by checking the functional equation (1.2), or by tailoring $L_{\mathfrak{p}}(Y, s)$ and $f_{\mathfrak{p}}$ in such a way that (1.2) holds. Of course, as long as (1.2) remains a conjecture, these methods are unable to prove correctness of the result.

In contrast, in this paper we compute $L_{\mathfrak{p}}(Y, s)$ and $f_{p}$ directly for all primes $\mathfrak{p}$ of bounded size (i.e. for $\mathrm{Np} \leq M$ for a certain constant $M$ ), for many curves $Y$ over $\mathbb{Q}$ of genus $g=2,3,4,5,6$. The computation at the primes of bad reduction is done using the methods of [6] and [15, and they are provably correct. We then verify the functional equation (1.2) numerically, using the methods of 8 .

The expected sign of the functional equation (1.2) is also known to be a product of local factors, the so called local root numbers (7]). In principal, it should be possible to compute the local root numbers using our methods, but we have not tried to do that. Of course, as a side effect of our numerical verification of the functional equation we obtain an experimental value for its sign, which is correct with very high probability.

We expect that with our approach it is now possible to compute examples with much larger conductor $N$ and genus $g$ than before. However, we have not tried to push computations to their limit. In our largest example, $g=6$ and $N=7 \cdot 11 \cdot 13 \cdot 89 \cdot 431 \cdot 857 \approx 3 \cdot 10^{10}$, which is comparable to the largest examples considered in 9 . 
1.3 In [6] we have shown how to compute the local factor $L_{\mathfrak{p}}(Y, s)$ and the conductor exponent $f_{\mathfrak{p}}$ from the stable reduction of $Y$ at $\mathfrak{p}$. Furthermore, we have shown how this can be done explicitly for superelliptic curves, i.e. curves $Y$ given by an equation of the form

$$
y^{n}=f(x),
$$

where $f \in K[x]$ is a polynomial with coefficients in $K$. A serious restriction that we imposed in [6] is that the exponent $n$ is prime to the residue characteristic of $\mathfrak{p}$. This restriction can be removed, using the results of [1] and [15]. So in principal we can compute $L_{\mathfrak{p}}(Y, s)$ and $f_{\mathfrak{p}}$ for all primes $\mathfrak{p}$ and all superelliptic curves. There is also no fundamental difficulty to extend our methods to curves which are not superelliptic. However, the details can get tricky, and it is rather hard to implement algorithms which work for general classes of curves.

The main class of examples we consider in the present paper is constructed in a way to illustrate our main point, while being at the same time as simple as possible and to be manageable by a straightforward algorithm. We consider a rather general family of hyperelliptic curves over $\mathbb{Q}$ of fixed genus $g_{Y} \geq 2$. Within this family we search for examples of curves $Y$ which have semistable reduction at every prime number $p$. For each curve satisfying this condition we compute its $L$-series and conductor and numerically verify the functional equation.

If a curve $Y$ does not have semistable reduction at a prime $\mathfrak{p}$, but only after replacing $K$ by a finite extension, the computation of $L_{\mathfrak{p}}(Y, s)$ and $f_{\mathfrak{p}}$ is much more involved. At the moment, we have not yet implemented algorithms which can handle such examples in a routine fashion. We discuss three examples in detail, illustrating the difficulties occurring. The discussion illustrates that each individual problem can typically be solved by a knowledgeable human supported by customized computational tools.

1.4 The structure of the paper is as follows. In $\S 2$ we recall how to compute the local $L$-factor and the conductor exponent at a prime $\mathfrak{p}$ where the curve $Y$ has semistable reduction. The explicit expression for the local $L$-factor (resp. the conductor exponent) can be found in Proposition 2.4 (resp. Corollary 2.5).

In $\S 3$ we consider a rather general class of hyperelliptic curves, and determine necessary and sufficient conditions for these curves to have semistable reduction everywhere (Lemmas 3.3 and 3.7). $\$ 3.5$ summarizes the algorithm for computing the local $L$-factor and the conductor exponent at the primes of bad reduction of the curves satisfying these conditions, and for verifying the functional equation numerically. Examples are given in $\S$ 3.6. In $\S$ three examples of superelliptic curves which do not have semistable reduction everywhere are discussed.

All data from the examples discussed in this paper can be retrieved from https: //www . uni-ulm.de/index .php?id=64504 


\section{2 Étale cohomology of a semistable curve}

Let $Y$ be a smooth projective and absolutely irreducible curve of genus greater than or equal to 2 defined over a number field $K$. In this section we recall from $\S 2$ of [6] the description of the local $L$-factor and the conductor exponent at a prime $\mathfrak{p}$ of $K$ in the case that $Y$ has semistable reduction at $\mathfrak{p}$. In general, the curve $Y$ only admits semistable reduction after passing to a finite extension. The main result of this section gives an explicit, computable expression for the local $L$-factor and the conductor exponent in the case that no field extension is needed.

2.1 For a (finite) prime $\mathfrak{p}$ of $K$ we write $\mathcal{O}_{\mathfrak{p}} \subset K$ for the local ring and $\mathbb{F}=\mathbb{F}_{\mathfrak{p}}$ for the residue field. Let $q:=\mathrm{Np}=|\mathbb{F}|$ denote the norm of $\mathfrak{p}$.

Throughout this section we assume that $Y$ has semistable reduction at $\mathfrak{p}$. Recall that this means that there exists a proper and flat model $\mathcal{Y}$ of $Y$ over $\mathcal{O}_{\mathfrak{p}}$ whose special fiber $\bar{Y}:=\mathcal{Y} \otimes_{\mathcal{O}_{\mathfrak{p}}} \mathbb{F}$ is semistable, i.e. $\bar{Y}$ is reduced and has only ordinary double points as singularities. We keep the semistable model $\mathcal{Y}$ fixed and call its special fiber $\bar{Y}$ the semistable reduction of $Y$ at $\mathfrak{p}$ (even though $\bar{Y}$ is not uniquely determined without further assumptions). We write $\bar{Y}_{k}:=\bar{Y} \otimes_{\mathbb{F}} k$ for the base change of $\bar{Y}$ to the algebraic closure $k$ of $\mathbb{F}$. We denote the absolute Galois group of $\mathbb{F}$ by $\Gamma_{\mathbb{F}}$. Let Frob $_{\mathfrak{p}} \in \Gamma_{\mathbb{F}}$ denote the arithmetic Frobenius element, i.e. the element determined by

$$
\operatorname{Frob}_{\mathfrak{p}}(a)=a^{q}
$$

for $a \in k$.

If $\bar{Y}$ is smooth (i.e. $\mathfrak{p}$ is a prime of good reduction) then it is well known that the local $L$-factor $L_{\mathfrak{p}}(Y, s)$ may be computed by point counting on $\bar{Y}$. Moreover, the conductor exponent is zero. In our case (where $\bar{Y}$ is semistable) this generalizes as follows. Let $H_{\mathrm{et}}^{i}\left(\bar{Y}_{k}, \mathbb{Q}_{\ell}\right)$ be the $i$ th $\ell$-adic étale cohomology group of $\bar{Y}_{k}$, where $\ell$ is an auxiliary prime different from the residue characteristic of p. Write $\operatorname{Frob}_{\bar{Y}}: \bar{Y} \rightarrow \bar{Y}$ for the relative $\mathbb{F}_{\mathfrak{p}}$-Frobenius morphism. For $n \in \mathbb{N}$ let $\mathbb{F}_{n} \subset k$ be the (unique) finite extension of $\mathbb{F}$ of degree $n$. The zeta function of $\bar{Y}$ is defined as

$$
Z(\bar{Y}, T):=\exp \left(\sum_{n \geq 1}\left|\bar{Y}\left(\mathbb{F}_{n}\right)\right| \cdot \frac{T^{n}}{n}\right) .
$$

It is well known that $Z(\bar{Y}, T)$ is a rational function of the form

$$
Z(\bar{Y}, T)=\frac{P_{1}(T)}{P_{0}(T) \cdot P_{2}(T)},
$$

where

$$
P_{i}(T):=\operatorname{det}\left(1-\left.T \cdot \operatorname{Frob}_{\bar{Y}}\right|_{H_{\mathrm{et}}^{i}\left(\bar{Y}_{k}, \mathbb{Q}_{\ell}\right)}\right) .
$$

See e.g. [14, Theorem 13.1. 
Proposition 2.1 The local L-factor is given by the formula

$$
L_{\mathfrak{p}}(Y / K, s)=P_{1}\left(q^{-s}\right)^{-1},
$$

where $P_{1}(T) \in \mathbb{Z}[T]$ is the numerator of the zeta function of $\bar{Y}$. The conductor exponent is

$$
f_{\mathfrak{p}}=2 g_{Y}-\operatorname{dim} H_{\mathrm{et}}^{1}\left(\bar{Y}_{k}, \mathbb{Q}_{\ell}\right)=2 g_{Y}-\operatorname{deg}\left(P_{1}\right) .
$$

Proof: This follows directly from [6], Corollaries 2.5 and 2.6, since we assume that $Y$ has semistable reduction over $K$.

Remark 2.2 Assuming we have an explicit equation for the curve $\bar{Y}$, Proposition 2.1 gives a simple way of computing $L_{\mathfrak{p}}(Y / K, s)$ via point counting. Indeed, it suffices to compute the polynomials $P_{i}$ for $i=0,1,2$. For $i=0,2$ this is easy. Since $\bar{Y}_{k}$ is connected we have $H_{\mathrm{et}}^{0}\left(\bar{Y}_{k}, \mathbb{Q}_{\ell}\right)=\mathbb{Q}_{\ell}$, with trivial action of $\operatorname{Frob}_{\bar{Y}}$ and hence

$$
P_{0}(T)=1-T .
$$

Let $\bar{Y}_{i}$ denote the irreducible components of $\bar{Y}$, and let $m_{i}$ denote the number of irreducible components of $\bar{Y}_{i} \otimes k$. Then

$$
P_{2}(T)=\prod_{i}\left(1-(q T)^{m_{i}}\right) .
$$

So in order to compute $P_{1}(T)$ (which has degree $\leq 2 g_{Y}$ ) it suffices to know the first $2 g_{Y}+1$ terms of the power series $Z(\bar{Y}, T)$, which depend on $\left|\bar{Y}\left(\mathbb{F}_{n}\right)\right|$ for $n=1, \ldots, 2 g_{Y}$.

Remark 2.3 If $\bar{Y}$ is smooth, the bound $2 g_{Y}$ from Remark 2.2 can be improved to $g_{Y}$, using the functional equation. More precisely, the polynomial $P_{1}(T)$ has the form

$$
P_{1}(T)=c_{0}+c_{1} T+\ldots+c_{2 g_{Y}} T^{2 g_{Y}} \in \mathbb{Z}[T],
$$

with $c_{0}=1$ and satisfies the functional equation

$$
P_{1}(1 / q T)=q^{-g_{Y}} T^{-2 g_{Y}} P_{1}(T),
$$

see e.g. 14, Theorem 12.6. The functional equation is equivalent to

$$
c_{2 g_{Y}-i}=q^{g_{Y}-i} c_{i}, \quad i=0, \ldots, g_{Y} .
$$

This means that $P_{1}$ is already determined by the coefficients $c_{0}, \ldots, c_{g_{Y}}$. It follows that $P_{1}(T)$ can be computed by counting the number of points of $\bar{Y}$ over the fields $\mathbb{F}_{n}$, for $n=1, \ldots, g_{Y}$. 
2.2 We have seen in the previous section that we can compute the local $L$-factor and the conductor exponent of $Y$ at a prime $\mathfrak{p}$ of semistable reduction, provided we know an explicit equation for the stable reduction $\bar{Y}$. In order to do this, we have to count the number of points of $\bar{Y}$ over certain finite extensions of the residue field of $\mathfrak{p}$. We note in passing that all the computations done for the present paper only use the naive counting method (as opposed to more sophisticated methods as e.g. in [11] or [10]).

If $\bar{Y}$ is smooth (i.e. if $Y$ has good reduction at $\mathfrak{p}$ ) we can use the functional equation to reduce the cost of point counting drastically (Remark 2.3). In this section we extend this trick to the case where $\bar{Y}$ is semistable. To keep the discussion simple, we assume that the curve $\bar{Y}$ is absolutely irreducible. This assumption is satisfied for our main class of examples considered in $\S$. As a first consequence we see that the denominator of the zeta function is of the most simple form,

$$
Z(\bar{Y}, T)=\frac{P(\bar{Y}, T)}{(1-T)(1-q T)} .
$$

Here $P(\bar{Y}, T)=P_{1}(T)$ in the notation of the previous subsection.

Let

$$
\pi: \bar{Y}_{0} \rightarrow \bar{Y}
$$

denote the normalization of $\bar{Y}$. Then $\bar{Y}_{0}$ is a smooth projective absolutely irreducible curve and $\pi$ is a finite birational morphism. If $\xi \in \bar{Y}$ is a closed point then the fiber $\pi^{-1}(\xi)$ has degree one over $\mathbb{F}(\xi)$ if $\xi$ is a smooth point and has degree two if $\xi$ is an ordinary double point. In the latter case, we say that $\xi$ is a split (resp. a nonsplit) double point if $\pi^{-1}(\xi)$ consists of two points (resp. of one point). Geometrically the map $\pi$ may be visualized as in Figure 2.1.

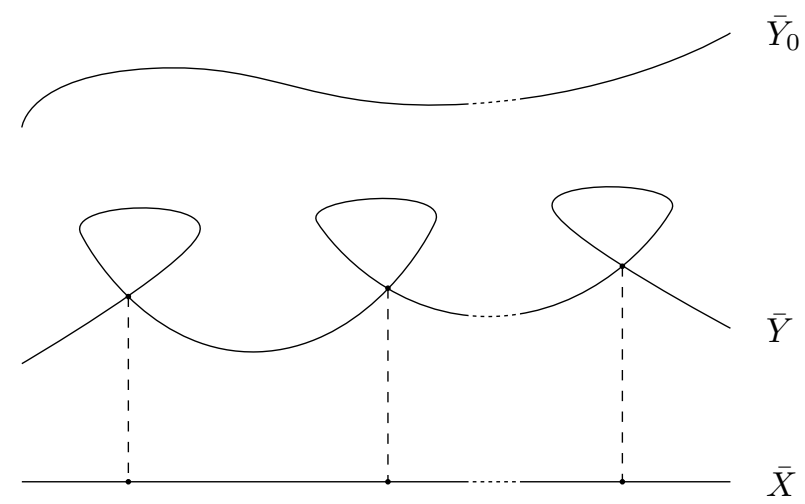

Figure 2.1: Normalization of $\bar{Y}$

Let $g_{0}$ denote the genus of $\bar{Y}_{0}$. The zeta function of $\bar{Y}_{0}$ has the form

$$
Z\left(\bar{Y}_{0}, T\right)=\frac{P\left(\bar{Y}_{0}, T\right)}{(1-T)(1-q T)},
$$


where $P\left(\bar{Y}_{0}, T\right)$ satisfies the functional equation, and hence can be determined by counting $\left|\bar{Y}_{0}\left(\mathbb{F}_{n}\right)\right|$ for $n=1, \ldots, g_{0}$ (Remark 2.3).

The following result reduces the calculation of the local $L$-factor in our situation to point counting on the normalization $\bar{Y}_{0}$ of $\bar{Y}$.

Proposition 2.4 Let $\mathcal{S}$ denote the set of singular points of $\bar{Y}$. For $\xi \in \mathcal{S}$ we let $d_{\xi}:=[\mathbb{F}(\xi): \mathbb{F}]$ denote the degree of $\xi$. Furthermore, define $\varepsilon_{\xi}:=1$ (resp. $\varepsilon_{\xi}:=-1$ ) if $\xi$ is a split (resp. a nonsplit) double point. Then

$$
P(\bar{Y}, T)=P\left(\bar{Y}_{0}, T\right) \cdot \prod_{\xi \in \mathcal{S}}\left(1-\varepsilon_{\xi} T^{d_{\xi}}\right) .
$$

Proof: Lemma 2.7.(1) of [6] implies that the $\ell$-adic étale cohomology group of $\bar{Y}$ decomposes as a direct sum of $\Gamma_{\mathbb{F}}$-modules

$$
H_{\mathrm{et}}^{1}\left(\bar{Y}_{k}, \mathbb{Q}_{\ell}\right)=H_{\mathrm{et}}^{1}\left(\bar{Y}_{0, k}, \mathbb{Q}_{\ell}\right) \oplus H^{1}\left(\Delta_{\bar{Y}_{k}}, \mathbb{Q}_{\ell}\right),
$$

where $\Delta_{\bar{Y}_{k}}$ denotes the graph of components of $\bar{Y}_{k}$. Therefore, it suffices to show that

$$
\operatorname{det}\left(1-\left.T \cdot \operatorname{Frob}_{\mathbb{F}}\right|_{H^{1}\left(\Delta_{\bar{Y}_{k}}, \mathbb{Q}_{\ell}\right)}\right)=\prod_{\xi \in \mathcal{S}}\left(1-\varepsilon_{\xi} T^{d_{\xi}}\right) .
$$

This amounts to computing the character of the representation of $\Gamma_{\mathbb{F}}$ acting on $H^{1}\left(\Delta_{\bar{Y}_{k}}, \mathbb{Q}_{\ell}\right)$, which is described in Lemma 2.7.(2) of [6].

Since we assume that $\bar{Y}$ is a semistable, absolutely irreducible curve, the graph $\Delta_{\bar{Y}_{k}}$ is a bouquet of $r$ circles, where

$$
r=\sum_{\xi \in \mathcal{S}} d_{\xi}
$$

is the number of ordinary double points of $\bar{Y}_{k}$ (see Figure2.1). An element $\xi \in \mathcal{S}$ corresponds to a $\Gamma_{\mathbb{F}^{-}}$orbit of edges of $\Delta_{\bar{Y}_{k}}$. Furthermore, $\xi$ is a split (resp. nonsplit) ordinary double point if and only if the stabilizer $\Gamma_{\mathbb{F}(\xi)}$ acts trivially (resp. acts by reversing orientation) on any of the edges in the orbit corresponding to $\xi$. Lemma 2.7.(2) of [6] implies that the character of $H^{1}\left(\Delta_{\bar{Y}_{k}}, \mathbb{Q}_{\ell}\right)$ considered as $\Gamma_{\mathbb{F}}$-representation is

$$
\chi_{\text {sing }}:=\bigoplus_{\xi \in \mathcal{S}} \operatorname{Ind}_{\Gamma_{\mathbb{F}(\xi)}^{\Gamma_{\mathbb{F}}} \varepsilon_{\xi}} .
$$

Here we interpret the integer $\varepsilon_{\xi} \in\{ \pm 1\}$ as the character of a 1-dimensional representation of $\Gamma_{\mathbb{F}(\xi)}$. Namely, $\varepsilon_{\xi}$ is the trivial character if $\varepsilon_{\xi}=1$ and the unique character of order 2 if $\varepsilon_{\xi}=-1$. The statement of the proposition now follows from an elementary calculation.

For a proof which does not use étale cohomology, see [3].

Corollary 2.5 The conductor exponent is

$$
f_{\mathfrak{p}}=r=\sum_{\xi \in \mathcal{S}} d_{\xi}
$$




\section{Hyperelliptic curves with semistable reduc- tion everywhere}

In this section we consider a class of hyperelliptic curves of genus greater than or equal to 2 which are defined over a number field $K$. We find conditions on the equation which guarantee that the curve has semistable reduction at every prime. This makes it relatively easy to calculate the local $L$-factor at the bad primes, even for residue characteristic $p=2$.

3.1 We fix a number field $K$, an integer $g_{Y} \geq 2$ and two polynomials $g, h \in$ $\mathcal{O}_{K}[x]$ satisfying the following three conditions:

- The polynomial $g$ is monic of degree $2 g_{Y}+1$.

- The degree of $h$ is at most $g_{Y}$.

- The polynomial $f:=4 g+h^{2}$ has no multiple roots.

Let $Y$ be the smooth projective curve over $K$ which is given birationally by the equation

$$
y^{2}+h(x) y=g(x) .
$$

By this we mean that the function field of $Y$ is the field extension of $K$ with two generators $x, y$ satisfying the above equation. Our assumptions imply that $Y$ is absolutely irreducible and, more specifically, a hyperelliptic curve of genus $g_{Y}$. An alternative equation for $Y$ is

$$
u^{2}=f(x):=4 g(x)+h(x)^{2},
$$

where $u:=2 y+h(x)$. Depending on the residue characteristic considered, either (3.1) or (3.2) will be more useful.

Equation (3.1) defines a smooth plane curve with a unique point 'at infinity' which we denote by $\infty$. It will be useful to have a similar equation for a neighborhood of the point $\infty$. For this we set $z:=x^{-1}, w:=z^{g_{Y}+1} y, g^{*}:=z^{2 g_{Y}+2} g$ and $h^{*}:=z^{g_{Y}+1} h$. Considering $g^{*}, h^{*}$ as polynomials in $z$, (3.1) can be rewritten as

$$
w^{2}+h^{*}(z) w=g^{*}(z)
$$

This is again an equation for a smooth plane curve, and the point $\infty$ has coordinates $(z, w)=(0,0)$. Note that we have used the assumption that $g$ has odd degree to prove smoothness at $\infty$.

3.2 We now choose a prime ideal $\mathfrak{p}$ of $\mathcal{O}_{K}$. Let $\mathcal{O}_{\mathfrak{p}}$ denote the local ring and $\mathbb{F}_{\mathfrak{p}}$ the residue field of $\mathfrak{p}$, as in $\S 2$

Let $\mathcal{X}=\mathbb{P}_{\mathcal{O}_{\mathfrak{p}}, x}^{1}$ be the projective line over $\mathcal{O}_{\mathfrak{p}}$ with coordinate $x$ and write $\mathcal{Y}$ for the normalization of $\mathcal{X}$ in the function field $K(Y)$ of $Y$. Then $\mathcal{Y}$ is a model of $Y$ over $\mathcal{O}_{\mathfrak{p}}$, i.e. $\mathcal{Y}$ is a flat and proper $\mathcal{O}_{\mathfrak{p}}$-scheme of finite type with generic fiber $Y$. 
We denote by $\bar{Y}$ and $\bar{X}$ the special fibers of $\mathcal{Y}$ and $\mathcal{X}$, respectively. These are proper curves over $\mathbb{F}_{\mathfrak{p}}$, and $\bar{X}=\mathbb{P}_{\mathbb{F}_{\mathfrak{p}}}$. We write $\bar{x}, \bar{y}$ for the image of $x, y$ in the function ring of $\bar{Y}$, and $\bar{g}$ (resp. $\bar{h}$ ) for the image of $g$ (resp. $h$ ) in $\mathbb{F}_{\mathfrak{p}}[\bar{x}]$. The following proposition shows that the curve $\bar{Y}$ is completely determined by the 'reduction' of (3.1) modulo $\mathfrak{p}$.

Proposition 3.1 The curve $\bar{Y}$ is reduced and absolutely irreducible. The point $\infty$ reduces to a smooth point $\bar{\infty} \in \bar{Y}$, and the affine open part $\bar{Y}-\{\bar{\infty}\}$ is a plane curve with equation

$$
\bar{y}^{2}+\bar{h}(\bar{x}) \bar{y}=\bar{g}(\bar{x}) .
$$

Proof: The curve $\mathcal{X}$ has an open affine covering $\left\{\operatorname{Spec} A_{1}\right.$, Spec $\left.A_{2}\right\}$, where $A_{1}=\mathcal{O}_{\mathfrak{p}}[x]$ and $A_{2}=\mathcal{O}_{\mathfrak{p}}[z]$. It follows that $\mathcal{Y}$ has an open affine covering $\left\{\right.$ Spec $B_{1}$, Spec $\left.B_{2}\right\}$, where $B_{i}$ is the integral closure of $A_{i}$ in $K(Y)=K(x, y)$. We claim that $B_{1}=A_{1}[y]$ and $B_{2}=A_{2}[w]$.

Let us first consider $B_{1}$. The minimal polynomial for $y$ over the function field $K(X)=K(x)$ is a monic polynomial with coefficients in $A_{1}$,

$$
F_{1}:=T^{2}+h T-g .
$$

It follows that $B_{1}^{\prime}:=A_{1}[y] \cong A_{1}[T] /\left(F_{1}\right)$ is finite and flat over $A_{1}$. Moreover, $B_{1}^{\prime} \otimes_{\mathcal{O}_{\mathfrak{p}}} K$ is integrally closed because (3.1) defines a smooth curve. Now

$$
B_{1} \otimes \mathbb{F}_{\mathfrak{p}}=\mathbb{F}_{\mathfrak{p}}[x, T] /\left(\bar{F}_{1}\right),
$$

where

$$
\bar{F}_{1}=T^{2}+\bar{h} T-\bar{g}
$$

is the image of $F_{1}$ in $\mathbb{F}_{\mathfrak{p}}[x, T]$. The polynomial $\bar{g} \in \mathbb{F}_{\mathfrak{p}}[x]$ still has odd degree $2 g_{Y}+1$ (because we have assumed that $g$ is monic). It follows that $\bar{F}_{1}$ is absolutely irreducible. We conclude using Lemma 4.1.18 of [13] that $B_{1}^{\prime}$ is integrally closed and hence $B_{1}=A_{1}[y]$. The proof that $B_{2}=A_{2}[w]$ is similar; one uses that (3.3) defines a smooth plane curve which remains reduced and irreducible after reduction to the residue field. The remaining statements are also easy to show.

3.3 We continue with the notation and assumptions of $\S \S 3.1$ and 3.2 . Additionally, we assume that the residue field $\mathbb{F}_{\mathfrak{p}}$ has characteristic $p=2$.

Notation 3.2 Let $\xi \in \bar{Y}-\{\bar{\infty}\}$ be a closed point and $\mathbb{F}_{p}(\xi)$ the residue field of $\xi$. We consider $\bar{Y}-\{\bar{\infty}\}$ as an affine plane curve with coordinate functions $\bar{x}, \bar{y}$. Set $a:=\bar{x}(\xi), b:=\bar{y}(\xi) \in \mathbb{F}_{p}(\xi)$. Then $\mathbb{F}_{\mathfrak{p}}(\xi)=\mathbb{F}_{\mathfrak{p}}(a, b)$, and we write $\xi=(a, b)$.

Lemma 3.3 Let $\xi=(a, b) \in \bar{Y}-\{\bar{\infty}\}$ be a closed point. 
(i) The point $\xi$ is a singularity of $\bar{Y}$ if and only if

$$
\bar{h}(a)=0=\left(\bar{h}^{\prime}(a)\right)^{2} \bar{g}(a)+\left(\bar{g}^{\prime}(a)\right)^{2} .
$$

Here $\bar{h}^{\prime}, \bar{g}^{\prime} \in \mathbb{F}_{\mathfrak{p}}[\bar{x}]$ are the formal derivatives of $\bar{h}, \bar{g}$ with respect to $\bar{x}$.

(ii) Assume that $\xi$ is a singularity. Then $\xi$ is an ordinary double point if and only if $\bar{h}^{\prime}(a) \neq 0$.

Proof: Clearly, $\xi=(a, b)$ satisfies (3.4):

$$
b^{2}+\bar{h}(a) b=\bar{g}(a) .
$$

The Jacobian criterion says that $\xi$ is singular if and only if

$$
\bar{h}(a)=0, \quad \bar{h}^{\prime}(a) b=\bar{g}^{\prime}(a) .
$$

Using (3.6) to eliminate $b$, we see that (3.6) is equivalent to (3.5). Now (i) is proved.

For the proof of (ii) we assume that $\xi$ is singular and compute the tangent cone of $\bar{Y}$ at $\xi$, using (3.6) and (3.7). We obtain

$$
(\bar{y}+b)^{2}+\bar{h}^{\prime}(a)(\bar{y}+b)(\bar{x}+a)+\bar{g}_{2}(\bar{x}+a)^{2}=0,
$$

where $\bar{g}_{2}$ is the coefficient of $\bar{x}^{2}$ in the Taylor expansion of $\bar{g}$ at $\bar{x}=a$. As we are in characteristic 2, the underlying quadratic form is nondegenerate if and only if $\bar{h}^{\prime}(a) \neq 0$. This proves (ii).

Corollary 3.4 The curve $\bar{Y}$ is semistable if and only if $\bar{h} \neq 0$ and

$$
\operatorname{gcd}\left(\bar{h}, \bar{h}^{\prime}, \bar{g}^{\prime}\right)=1 .
$$

Proof: To prove the corollary it suffices to show that $\xi=(a, b) \in \bar{Y}-\{\bar{\infty}\}$ is a smooth or an ordinary double point if and only if $\left(\bar{h}(a), \bar{h}^{\prime}(a), \bar{g}^{\prime}(a)\right) \neq(0,0,0)$. This follows directly from Lemma 3.3 .

From now on we assume that $\bar{Y}$ is semistable, and we use the results from $\S 2$ to compute the local $L$-factor and the conductor exponent of $Y$ at $\mathfrak{p}$. Let

$$
\pi: \bar{Y}_{0} \rightarrow \bar{Y}
$$

be the normalization of $\bar{Y}$. Recall that $\pi$ is a finite map which is an isomorphism above the smooth locus of $\bar{Y}$ (as in Figure 2.1). In order to use Proposition 2.4 and Corollary 2.5 we need to know the set $\mathcal{S}$ of singular points of $\bar{Y}$, the invariants $d_{\xi}$ and $\varepsilon_{\xi}$, for all $\xi \in \mathcal{S}$, and an explicit equation for $\bar{Y}_{0}$. This will be achieved by the following proposition and Corollary 3.6 .

Proposition 3.5 Assume that $\bar{Y}$ is semistable. Set

$$
r:=\operatorname{gcd}\left(\bar{h},\left(\bar{h}^{\prime}\right)^{2} \bar{g}+\left(\bar{g}^{\prime}\right)^{2}\right) \in \mathbb{F}[\bar{x}] .
$$

Then the following holds. 
(i) A point $\xi \in \bar{Y}-\{\bar{\infty}\}$ is singular if and only if $r(a)=0$.

(ii) The polynomial $r$ is separable, i.e. all roots of $r$ over the algebraic closure $k$ of $\mathbb{F}$ are simple. Furthermore, $\tilde{h}:=\bar{h} / r \in \mathbb{F}[\bar{x}]$ is prime to $r$.

(iii) There exists $s \in \mathbb{F}[\bar{x}]$ such that

$$
r^{2} \mid \bar{g}+s^{2}+\bar{h} s
$$

(iv) Set $\tilde{g}:=\left(\bar{g}+s^{2}+\bar{h} s\right) / r^{2} \in \mathbb{F}[\bar{x}]$ and $\tilde{y}:=(\bar{y}+s) / r \in \mathbb{F}(\bar{Y})$. Then $\tilde{y}$ satisfies

$$
\tilde{y}^{2}+\tilde{h} \tilde{y}=\tilde{g},
$$

which is an equation for the smooth plane affine $\bar{Y}_{0}-\{\bar{\infty}\}$.

Proof: (i) follows directly from Lemma 3.3 (i). Now assume that $a$ is a root of $r$. Then there is a unique point $\xi=(a, b) \in \bar{Y}$, and it is a singularity. Since we assume that $\bar{Y}$ is semistable, $\xi$ is even an ordinary double point. Therefore, it follows from Lemma 3.3 (ii) that $\bar{h}^{\prime}(a) \neq 0$. We conclude that all roots of $r$ are simple roots of $\bar{h}$. This proves (ii).

Since $\mathbb{F}$ is a perfect field of characteristic 2 and $r$ is separable by (ii), there exists a polynomial $s \in \mathbb{F}[\bar{x}]$ such that

$$
s^{2} \equiv \bar{g} \quad(\bmod r) .
$$

Set $\tilde{y}:=(\bar{y}+s) / r \in \mathbb{F}(\bar{Y})$. Then $\tilde{y}$ satisfies equation (3.8).

For the proof of (iii) we have to show that $\tilde{g}$ is a polynomial. Assume that $a \in k$ is a pole of $\tilde{g}$. By (ii) $r$ has a simple zero at $a$. The choice of $s$ implies that $\bar{h}$ also has a simple zero at $a$, and hence that $\tilde{g}$ has a simple pole at $\bar{x}=a$. But this would mean that the map $\bar{Y}_{0} \rightarrow \bar{X}=\mathbb{P}_{\mathbb{F}}^{1}$ is branched at $\bar{x}=a$. This would imply that there exists a unique smooth point $\xi=(a, b) \in \bar{Y}$ above $\bar{x}=a$, contradicting the fact that $r(a)=0$. Now (iii) is proved.

It follows from (iii) that there is a finite birational morphism $\bar{Y}_{1} \rightarrow \bar{Y}$ which is an isomorphism at $\bar{\infty}$ and such that $\bar{Y}_{1}-\{\bar{\infty}\}$ is the plane affine curve given by (3.8). Let $\xi=(a, b) \in \bar{Y}_{1}-\{\bar{\infty}\}$ be a closed point. If $\xi$ is a singular point, then $\tilde{h}(a)=0$ by the Jacobian criterion. But then $r(a) \neq 0$ by (ii) and the definition of $\tilde{h}$. Therefore, $\xi$ lies above a smooth point of $\bar{Y}$. Since $\bar{Y}_{1} \rightarrow \bar{Y}$ is finite, it follows that $\xi$ is a smooth point as well, contradiction. We conclude that $\bar{Y}_{1}$ is smooth. This implies that $\bar{Y}_{1}=\bar{Y}_{0}$ is the normalization of $\bar{Y}$ and completes the proof of the proposition.

Corollary 3.6 Assume that $\bar{Y}$ is semistable.

(i) There is a bijection between the set $\mathcal{S}$ of singular points of $\bar{Y}$ and the irreducible factors of the polynomial $r \in \mathbb{F}[\bar{x}]$ defined in Proposition 3.5 .

(ii) A singular point $\xi=(a, b) \in \bar{Y}$ is a split (resp. a non split) ordinary double point if the polynomial

$$
T^{2}+\tilde{h}(a) T+\tilde{g}(a) \in \mathbb{F}[T]
$$

is reducible (resp. irreducible). 
(iii) The conductor exponent at $\mathfrak{p}$ is

$$
f_{\mathfrak{p}}=\operatorname{deg}(r) .
$$

3.4 We now switch to the case of a prime $\mathfrak{p}$ with residue characteristic $p \geq 3$. It will be more convenient to use Equation (3.2) to describe the curve $Y$ :

$$
u^{2}=f(x):=4 g(x)+h(x)^{2} .
$$

Recall that this equation is derived from (3.1) by the substitution $y=(u-h) / 2$. Since 2 is a unit in $\mathcal{O}_{\mathfrak{p}}$, the same substitution works for the model $\mathcal{Y}$. It follows that the special fiber $\bar{Y}$ of $\mathcal{Y}$ is given by the equation

$$
\bar{u}^{2}=\bar{f}(\bar{x}) .
$$

Here $\bar{f} \in \mathbb{F}_{\mathfrak{p}}[\bar{x}]$ denotes the image of $f$ in $\mathbb{F}_{\mathfrak{p}}[\bar{x}]$ and $\bar{u}$ the image of $u$ in $\mathbb{F}(\bar{Y})$. We also adopt Notation 3.2 to this new equation and write a closed point $\xi \in$ $\bar{Y}-\{\bar{\infty}\}$ in the form $\xi=(a, b)$, where $(a, b)$ is a solution to (3.9).

Note that by choice of $g$ (monic, degree $2 g_{Y}+1$ ) and $h$ (degree $\leq g_{Y}$ ), both $f$ and $\bar{f}$ have degree $2 g_{Y}+1$. The polynomial $f$ is separable by assumption, but in general this will not be true for $\bar{f}$. The following is very easy to show.

Lemma 3.7 The curve $\bar{Y}$ is semistable if and only if $\bar{f}$ has at most double roots.

Let us assume from now on that the curve $\bar{Y}$ is semistable. By Lemma 3.7 the polynomial $\bar{f}$ has at most double roots. It follows that there is a unique decomposition

$$
\bar{f}=r^{2} \cdot s,
$$

where $r, s \in \mathbb{F}[\bar{x}]$ are separable and relatively prime.

Proposition 3.8 We assume that $\bar{Y}$ is semistable. Let $\xi=(a, b) \in \bar{Y}-\{\bar{\infty}\}$ be a closed point.

(i) The point $\xi$ is a singularity of $\bar{Y}$ if and only if $r(a)=0$.

(ii) Assume $\xi$ is a singularity. Then $\xi$ is a split (resp. a non split) ordinary double point if and only if $s(a)$ is a square (resp. a nonsquare) in $\mathbb{F}^{\times}$.

(iii) The normalization $\bar{Y}_{0}$ of $\bar{Y}$ is given by the equation

$$
\bar{v}^{2}=s(\bar{x}) .
$$

The map $\bar{Y}_{0} \rightarrow \bar{Y}$ is determined by $\bar{u}=r \bar{v}$.

Proof: The proof is similar to but easier than the proof of Proposition 3.5. and is therefore omitted.

Corollary 3.9 Assume that $\bar{Y}$ is semistable. 
(i) There is a bijection between the set $\mathcal{S}$ of singular points of $\bar{Y}$ and the irreducible factors of the polynomial $r$.

(ii) For $\xi=(a, b) \in \mathcal{S}$ we have $\varepsilon_{\xi}=1$ (resp. $\varepsilon_{\xi}=-1$ ) if and only if $s(a)$ is a square (resp. a nonsquare) in $\mathbb{F}_{\mathfrak{p}}^{\times}$.

(iii) The conductor exponent is

$$
f_{\mathfrak{p}}=\operatorname{deg}(r)
$$

3.5 In this section we summarize the results obtained so far and describe the resulting algorithm for computing the $L$-function of the curve $Y$. We also make some comments on the implementation and running time. For simplicity we assume from now on that $K=\mathbb{Q}$.

We are interested in computing a certain $L$-series given as an Euler product,

$$
L(Y / K, s)=\sum_{n \geq 1} \frac{a_{n}}{n^{s}}=\prod_{p} L_{p}(Y, s) .
$$

More specifically, we want to give evidence for the conjectured functional equation (Equation (1.2)). We use the Dokchitser package in the free computer algebra software sage, based on Tim Dokchitser's paper [8. To verify the functional equation in this package, we need to know the conductor of the $L$-function,

$$
N=\prod_{p} p^{f_{p}}
$$

and the coefficients $a_{n}$ need to be calculated for all $n \leq M$ up to a certain heuristic constant $M$, depending on $N$ and $g_{Y}$. The constant $M$ can be computed using the Dokchitser package. Due to the multiplicativity relation of the $a_{n}$, we only have to compute the coefficients $a_{p^{k}}$ for prime powers $p^{k} \leq M$ via point counting. If one uses naive point counting methods, the calculation of each $a_{p^{k}}$ has a complexity of about $\mathcal{O}\left(p^{k}\right)$. By the prime number theorem, we get a complexity of $\mathcal{O}(M / 2 \cdot M / \log (M))$ for each $L$-series. For fixed genus, $M$ is proportional to the square root of the conductor $N$ of the curve (cf. 8 ). So the complexity of checking the functional equation is bounded by $\mathcal{O}(N / \log N)$.

Finding examples of suitable curves $Y$ and checking the functional equation of $L(Y, s)$ can be performed as follows. We fix an integer $g_{Y} \geq 2$.

1. Choose polynomials $g, h \in \mathbb{Z}[x]$ with $h \not \equiv 0(\bmod 2), g$ monic, $\operatorname{deg}(g)=$ $2 g_{Y}+1, \operatorname{deg}(h) \leq g_{Y}$. Consider the reductions of $g, h$ modulo 2 . If

$$
\operatorname{gcd}\left(\bar{h}, \bar{h}^{\prime}, \bar{g}^{\prime}\right) \neq 1,
$$

the model $\mathcal{Y}$ from $\S 3.2$ is not semistable at $p=2$ (Corollary 3.4). In this case we dismiss our choice of $g$ and $h$ and start over again. Otherwise, compute the polynomial

$$
r:=\operatorname{gcd}\left(\bar{h},\left(\bar{h}^{\prime}\right)^{2} \bar{g}+\left(\bar{g}^{\prime}\right)^{2}\right) .
$$


2. Calculate the discriminant $\Delta \in \mathbb{Z}$ of the polynomial $f:=4 g+h^{2}$, and define $S^{\prime}$ as the set of prime factors of $\Delta$, ignoring the prime factor 2 . Check for all $p \in S^{\prime}$ whether

$$
\operatorname{gcd}\left(\bar{f}, \bar{f}^{\prime}, \bar{f}^{\prime \prime}\right)=1 .
$$

If the test fails for one $p \in S^{\prime}$ then we cannot guarantee that $Y$ has semistable reduction (Lemma 3.7). If this happens we dismiss our example and go back to the beginning. If $\operatorname{deg} r>1$ then we set $S:=S^{\prime} \cup\{2\}$, otherwise set $S:=S^{\prime}$.

Now we know that $Y$ has bad semistable reduction at all primes $p \in S$ and good reduction everywhere else.

3. For all bad primes $p \in S$, we do the following.

3a. For $p=2$, decompose the polynomial $r \in \mathbb{F}_{2}[\bar{x}]$ into irreducible factors,

$$
r=\prod_{i} r_{i} .
$$

Each factor $r_{i}$ corresponds to a singularity $\xi_{i} \in \bar{Y}$ with $\operatorname{deg}\left(\xi_{i}\right)=$ $\operatorname{deg}\left(r_{i}\right)$. Check for all $i$ whether $\xi_{i}$ is split or not (Corollary [3.6 (ii)) and set $\varepsilon_{i} \in\{ \pm 1\}$ accordingly.

Now calculate the numerator $P\left(\bar{Y}_{0}, T\right)$ of the zeta function of the normalization $\bar{Y}_{0}$ of $\bar{Y}$, using Equation (3.8) and point counting (Remark 2.2 and Remark 2.3). The local $L$-factor at $p=2$ is

$$
L_{2}(\bar{Y}, T)=P\left(\bar{Y}_{0}, T\right)^{-1} \prod_{i}\left(1-\varepsilon_{i} T^{d_{i}}\right)^{-1},
$$

see Proposition 2.4. Also, $f_{2}=\operatorname{deg}(r)$ (Corollary 3.6 (iii)).

3b. For $p \in S-\{2\}$, compute the decomposition

$$
\bar{f}=r^{2} s .
$$

Split $r=\prod_{i} r_{i} \in \mathbb{F}_{p}[x]$ into irreducible factors. Set $d_{i}:=\operatorname{deg}\left(r_{i}\right)$ and $\varepsilon_{i}:= \pm 1$, according to Corollary 3.9 (ii).

Calculate the numerator $P\left(\bar{Y}_{0}, T\right)$ of the zeta function of the normalization $\bar{Y}_{0}$ of $\bar{Y}$, using the equation from Proposition 3.8 (iii). As in 3a, the local $L$-factor is

$$
L_{p}(\bar{Y}, T)=P\left(\bar{Y}_{0}, T\right)^{-1} \prod_{i}\left(1-\varepsilon_{i} T^{d_{i}}\right)^{-1} .
$$

Set $f_{p}:=\operatorname{deg}(r)$ (Corollary 3.9 (iii)).

4. Compute the conductor

$$
N:=\prod_{p \in S} p^{f_{p}}
$$

and the constant $M$. 
5. Calculate the local $L$-factor $L_{p}(Y, s)$ for all good primes $p \notin S, p \leq M$ via point counting (Remarks 2.2 and 2.3).

6. Compute the truncated $L$-series

$$
L(Y, s)^{\prime}=\sum_{n=1}^{M} \frac{a_{n}}{n^{-s}}
$$

from the Euler factors $L_{p}(Y, s), p \leq M$. Check the functional equation using the Dokchitser package for the root number 1. If this fails, repeat with root number -1 .

Remark 3.10 The algorithm described above can be slightly improved as follows. Observe that we only need coefficients $a_{n}$ of the $L$-series with $n \leq M$. Thus we can use the bound $M$ (which only depends on the conductor exponents $f_{p}$ for $\left.p \in S\right)$ to truncate the polynomial $P\left(\bar{Y}_{0}, T\right)$, resp. the local $L$-factor $L_{p}$, in order to avoid counting points over fields with more than $M$ elements. This affects the computation of the polynomials $P\left(\bar{Y}_{0}, T\right)$ in Step 3a and 3b. For an example, we refer to Example 3.14 in $\S 3.6$.

In the above algorithm, the time needed to compute the set of bad primes, the conductor $N$ and the constant $M$ is insignificant compared to the time needed for the point counting. The numerical verification of the functional equation is not expensive either. Therefore, the running time of our algorithm for an individual curve $Y$ is indeed bounded by $\mathcal{O}(N / \log N)$, as explained above, with almost all the running time spent on point counting. For the class of hyperelliptic curves considered in this section, examples with conductor up to $10^{10}$ can be computed within a reasonable time. In the largest example that we computed, the conductor is $N=7 \cdot 11 \cdot 13 \cdot 89 \cdot 431 \cdot 857 \approx 3 \cdot 10^{10}$. Using more sophisticated point-counting methods as e.g. in [12] would probably allow the computation of significantly larger examples.

The running time of our example is essentially determined by the conductor. Although the constant $M$ depends on $N$ and the genus of $Y$, its dependence on $g_{Y}$ is insignificant within the range of genera that we consider (an asymptotic estimate for $M$ can be obtained from [8], $\S 4$, in particular Equation (4-2)). This is an advantage of our approach, as opposed to for example that of Booker (4), $\S 2.3 .2)$. However, the discriminant of the polynomial $f=4 g+h^{2}$ determines the odd prime factors of the conductor. Thus with growing degree of $g$, and therefore with growing genus $g_{Y}=(\operatorname{deg}(g)-1) / 2$, it gets more and more difficult to find examples of curves $Y$ with conductor of reasonable size. So far, we managed to find examples that fall within this range for all $g_{Y} \leq 6$.

We have verified the functional equation for several hundreds of examples. Obviously, one can easily construct a lot more examples, especially for small genus. On our homepage, we provide a selection of examples - each with slightly different parameters - where the functional equation has been verified. The data can be found on https://www. uni-ulm.de/index.php?id=64504. 
3.6 In this section we give a few explicit examples, in detail. All given examples have been checked to fulfill the functional equation. Note that the chosen examples do not necessarily have the smallest possible conductor for the given genus - it is merely a selection of 'typical' examples.

Example 3.11 The polynomials

$$
g=x^{5}-3 x^{4}-3 x^{3}-3 x^{2}-3 x-1, \quad h=x^{2}+3 x+1
$$

define a genus-two curve $Y / \mathbb{Q}$. We find five bad primes: $2,3,7,101,163$. The $L$-factors corresponding to these primes are (we write $T$ instead of $p^{-s}$ ):

$$
\begin{aligned}
L_{2}^{-1} & =1+T^{2}, \\
L_{3}^{-1} & =(1+T)\left(3 T^{2}-T+1\right), \\
L_{7}^{-1} & =(1-T)\left(7 T^{2}+3 T+1\right), \\
L_{101}^{-1} & =(1+T)\left(101 T^{2}+3 T+1\right), \\
L_{163}^{-1} & =(1-T)\left(163 T^{2}+11 T+1\right) .
\end{aligned}
$$

The conductor is $N=2^{2} \cdot 3 \cdot 7 \cdot 101 \cdot 163 \approx 10^{6}$. We briefly review the computations for $p=2,3$. For $p=2$ we look at the curve

$$
\bar{Y} / \mathbb{F}_{2}: \bar{y}^{2}+\left(1+\bar{x}+\bar{x}^{2}\right) \bar{y}=1+\bar{x}+\bar{x}^{2}+\bar{x}^{3}+\bar{x}^{4}+\bar{x}^{5} .
$$

Since $\bar{h}^{\prime}=\left(1+\bar{x}+\bar{x}^{2}\right)^{\prime}=1, \bar{Y}$ is semistable. The singular locus is determined by the polynomial

$$
r:=\operatorname{gcd}\left(\bar{h},\left(\bar{h}^{\prime}\right)^{2} \bar{g}+\left(\bar{g}^{\prime}\right)^{2}\right)=\bar{h}=1+\bar{x}+\bar{x}^{2} .
$$

Hence there is a unique ordinary double point $\xi=(a, b)$ of degree 2, where $a$ is a solution to $a^{2}+a+1=0$. Substituting $\bar{y}=\bar{h} \tilde{y}$ into the equation for $\bar{Y}$ and dividing by $\bar{h}^{2}$ we obtain the equation for its normalization:

$$
\tilde{y}^{2}+\tilde{y}=\bar{g} / \bar{h}^{2}=1+\bar{x} .
$$

This is a curve of genus zero, so it doesn't contribute to the local $L$-factor. However, we can see that the inverse image $\pi^{-1}(\xi)$ of the singular point $\xi$ corresponds to the solutions to the equation

$$
\tilde{y}^{2}+\tilde{y}=1+a
$$

in $\mathbb{F}_{2}(a)=\mathbb{F}_{4}$. Clearly, this equation is irreducible and so $\xi$ is a non-split ordinary double point. Therefore,

$$
L_{2}(Y, s)=\frac{1}{1+2^{-2 s}} .
$$

For $p=3$ we are looking at the curve

$$
\bar{Y} / \mathbb{F}_{3}: \bar{u}^{2}=\bar{x}^{2}\left(2+\bar{x}^{2}+\bar{x}^{3}\right) .
$$


This is a semistable curve with one $\mathbb{F}_{3}$-rational ordinary double point $\xi=(0,0)$. Substituting $\bar{u}=\bar{x} \bar{v}$ and dividing by $\bar{x}^{2}$ gives an equation for the normalization of $\bar{Y}$,

$$
\bar{Y}_{0}: \bar{v}^{2}=2+\bar{x}^{2}+\bar{x}^{3},
$$

a smooth curve of genus 1 over $\mathbb{F}_{3}$. There are exactly three rational points, $\left|\bar{Y}_{0}\left(\mathbb{F}_{3}\right)\right|=3$. It follows that the numerator of the zeta function is $P\left(\bar{Y}_{0}, T\right)=$ $1-T+3 T^{2}$. Also, the fiber $\pi^{-1}(\xi)$ is given by the equation

$$
\bar{v}^{2}=2,
$$

which is irreducible over $\mathbb{F}_{3}$. It follows that $\xi$ is a non-split double point and that

$$
L_{3}(Y, s)=\frac{1}{\left(1+3^{-s}\right)\left(1-3^{-s}+3^{1-2 s}\right)} .
$$

The computation of the $L$-factors for $p=7,101,163$ is similar.

The numerical verification of the functional equation was successful, with root number 1 .

Example 3.12 The polynomials $g=x^{7}+x^{6}+2 x^{5}+2 x^{4}+2 x^{3}-1$ and $h=$ $-x^{3}+x^{2}+x+2$ define a genus-three curve. We find four bad primes: $2,3,11,37$. The $L$-factors corresponding to these primes are

$$
\begin{aligned}
& L_{2}^{-1}=(1-T)(1+T)\left(2 T^{2}-T+1\right), \\
& L_{3}^{-1}=(1+T)\left(1-T^{2}\right), \\
& L_{11}^{-1}=(1+T)^{2}\left(11 T^{2}-4 T+1\right), \\
& L_{37}^{-1}=(1-T)\left(37^{2} T^{4}+148 T^{3}+14 T^{2}+4 T+1\right),
\end{aligned}
$$

the conductor is $N=2^{2} \cdot 3^{3} \cdot 11^{2} \cdot 37 \approx 10^{5}$, and the root number is 1 .

Example 3.13 The polynomials $g=x^{9}-2 x^{8}+x^{7}-2 x^{4}+2 x^{3}+2 x^{2}+x$ and $h=-2 x^{4}+x^{3}-2 x^{2}-x-1$ define a genus-four curve. We find four bad primes: $3,7,31,53$. The $L$-factors corresponding to these primes are

$$
\begin{aligned}
& L_{3}^{-1}=(1-T)(1+T)\left(9 T^{4}+6 T^{3}+4 T^{2}+2 T+1\right), \\
& L_{7}^{-1}=\left(1+T^{3}\right)\left(7 T^{2}+3 T+1\right), \\
& L_{31}^{-1}=(1-T)\left(31^{3} T^{6}+1581 T^{4}+36 T^{3}+51 T^{2}+1\right), \\
& L_{53}^{-1}=(1+T)\left(53^{3} T^{6}+8427 T^{5}+1537 T^{4}+670 T^{3}+29 T^{2}+3 T+1\right),
\end{aligned}
$$

the conductor is $N=3^{2} \cdot 7^{3} \cdot 31 \cdot 53 \approx 10^{8}$, and the root number is 1 .

Example 3.14 The polynomials $g=x^{11}+3 x^{4}+2 x^{3}-3 x^{2}-2 x$ and $h=-3 x^{3}+$ $x^{2}+3 x+1$ define a genus-five curve. We find four bad primes: 7, 227, 1277, 1609 . 
The truncated $L$-factors corresponding to these primes are

$$
\begin{aligned}
L_{7}^{-1} & =(1-T)\left(7^{4} T^{8}-588 T^{6}+134 T^{4}-12 T^{2}+1\right), \\
L_{227}^{-1} & =(1+T)\left(\ldots+200 T^{2}+13 T+1\right), \\
L_{1277}^{-1} & =(1+T)(\ldots-35 T+1), \\
L_{1609}^{-1} & =(1+T)(\ldots-26 T+1) .
\end{aligned}
$$

The conductor is $N=7 \cdot 227 \cdot 1277 \cdot 1609 \approx 10^{9}$, and the root number is 1 . We truncated the last three $L$-factors to save computation time, since the bound in this example is $M=1112661<\left\{227^{3}, 1277^{2}, 1609^{2}\right\}$. Hence no further information is needed to verify the functional equation $(\oint 3.5)$.

Example 3.15 The polynomials $g=x^{13}+x^{12}+x^{11}+x^{10}+x^{9}+x^{8}-x^{7}-x^{6}+$ $x^{5}+x^{4}+x^{3}$ and $h=x^{6}+x^{5}-x^{3}+x^{2}+x+1$ define a genus-six curve. We find six bad primes: $7,11,13,89,431,857$. The truncated $L$-factors corresponding to these primes are

$$
\begin{aligned}
L_{7}^{-1}= & (1+T)\left(7^{5} T^{10}+1372 T^{8}+1127 T^{7}+112 T^{6}+122 T^{5}+16 T^{4}+23 T^{3}+\right. \\
& \left.4 T^{2}+1\right) \\
L_{11}^{-1}= & (1+T)\left(11^{5} T^{10}-43923 T^{9}-10648 T^{8}+2662 T^{7}+781 T^{6}-390 T^{5}+\right. \\
& \left.71 T^{4}+22 T^{3}-8 T^{2}-3 T+1\right) \\
L_{13}^{-1}= & (1+T)\left(13^{5} T^{10}-114244 T^{9}+43940 T^{8}-10140 T^{7}+1040 T^{6}+\right. \\
& \left.-342 T^{5}+80 T^{4}-60 T^{3}+20 T^{2}-4 T+1\right), \\
L_{89}^{-1}= & (1-T)\left(\ldots+320 T^{3}+43 T^{2}-5 T+1\right), \\
L_{431}^{-1}= & (1+T)\left(\ldots+859 T^{2}+31 T+1\right) \\
L_{857}^{-1}= & (1-T)\left(\ldots+1448 T^{2}-41 T+1\right)
\end{aligned}
$$

The conductor is $N=7 \cdot 11 \cdot 13 \cdot 89 \cdot 431 \cdot 857 \approx 3 \cdot 10^{10}$, and the root number is 1 . As in the previous example, we truncated the last three $L$-factors, for the bound is $M=2549728<\left\{89^{4}, 431^{3}, 857^{3}\right\}$.

\section{More examples}

Combining the results of [6], 2], [1] and [15] it is in principle possible to compute the semistable reduction of any superelliptic curve and therefore the local $L$ factors and the conductor exponents, at all primes. In the previous section we have chosen a class of examples where this was particularly easy. In this section we treat a small sample of examples which do not fall within this class, but where we were nevertheless able to compute $L_{p}(Y, s)$ and $f_{p}$ for all $p$ and to verify the functional equation. 
4.1 The polynomials

$$
g=x^{7}-2 x^{6}-2 x^{4}+x^{3}+3 x^{2}+x, \quad h=3 x^{3}+3 x^{2}+2 x+1
$$

substituted in (3.1) define a genus-three hyperelliptic curve $Y / \mathbb{Q}$. One checks that $Y$ has good reduction at $p=2$ (see Step 1 and 2 in the algorithm from $\S$ 3.5). We ignore the prime $p=2$ from now on and use the equation

$$
Y / \mathbb{Q}: y^{2}=f(x)=4 x^{7}+x^{6}+18 x^{5}+13 x^{4}+22 x^{3}+22 x^{2}+8 x+1,
$$

with $f:=h^{2}+4 g$, to describe $Y$. (As in $\S 3$ this means that the open affine subset $Y-\{\infty\} \subset Y$ is the plane affine curve given by the above equation.) The discriminant of $f$ is $\Delta=-2^{12} \cdot 3 \cdot 5^{3} \cdot 13^{2} \cdot 97$. Therefore, there are four bad primes: $p=3,5,13,97$. For the primes $p=3,13,97$ the condition $\operatorname{gcd}\left(\bar{f}, \bar{f}^{\prime}, \bar{f}^{\prime \prime}\right)=1$ holds and therefore $Y$ has semistable reduction at $p$. The local $L$-factors and the conductor exponent can be computed as before. We obtain

$$
\begin{aligned}
& L_{3}^{-1}=(1-T)\left(3^{2} T^{4}+3 T^{3}+T+1\right), \quad f_{3}=1, \\
& L_{13}^{-1}=\left(1+T^{2}\right)\left(13 T^{2}+5 T+1\right), \quad f_{13}=2, \\
& L_{97}^{-1}=(1+T)\left(97^{2} T^{4}+582 T^{3}+78 T^{2}+6 T+1\right), \quad f_{97}=1 .
\end{aligned}
$$

However, for $p=5$ the special fiber of the naive model $\mathcal{Y}^{\text {naive }}$ of $Y$ over $\mathbb{Z}_{(5)}$ (obtained by reducing equation (4.1) modulo 5) is the curve

$$
\bar{Y}^{\text {naive }} / \mathbb{F}_{5}: \bar{y}^{2}=\bar{f}=4(\bar{x}+1)^{4}\left(\bar{x}^{3}+\bar{x}+4\right) .
$$

We see that $\bar{Y}^{\text {naive }}$ has a unique $\mathbb{F}_{5}$-rational singularity $(\bar{x}, \bar{y})=(4,0)$ which is not an ordinary double point, and is smooth everywhere else. In particular, $\bar{Y}^{\text {naive }}$ is not semistable, and the methods from $\S 3$ are not directly applicable.

Nevertheless, using the results of [6] we can easily compute the semistable reduction of $Y$ at $p=5$. We are dealing with a local problem and may therefore consider $Y$ as a curve over the 5 -adic numbers $\mathbb{Q}_{5}$. Let $L:=\mathbb{Q}_{5}[\pi]$ be the extension of degree 4 with $\pi^{4}=5$. Clearly $L / \mathbb{Q}_{5}$ is a Galois extension, which is totally and tamely ramified. The Galois group of $L / \mathbb{Q}_{5}$ is cyclic, generated by the element $\sigma$ determined by

$$
\sigma(\pi)=\zeta_{4} \pi .
$$

Here $\zeta_{4} \in \mathbb{Z}_{5}$ is the 4 th root of unity with $\zeta_{4} \equiv 2(\bmod 5)$. Let $\mathfrak{p}=(\pi) \triangleleft \mathcal{O}_{L}$ denote the unique prime ideal. Note that the residue field is $\mathbb{F}_{\mathfrak{p}}=\mathbb{F}_{5}$.

Lemma 4.1 (i) The curve $Y_{L}=Y \otimes_{\mathbb{Q}_{5}} L$ has semistable reduction at $\mathfrak{p}$.

(ii) Let $\mathcal{Y} / \mathcal{O}_{L}$ denote the minimal semistable model of $Y_{L}$ and $\bar{Y}$ its special fiber. The curve $\bar{Y}$ is the union of two smooth, absolutely irreducible curves over $\mathbb{F}_{5}$. The first component $\bar{Y}_{1}$ has an affine open subset which is given by the equation

$$
\bar{y}_{1}^{2}=4\left(\bar{x}^{3}+\bar{x}+4\right)
$$


the second component $\bar{Y}_{2}$ has an affine open subset given by

$$
\bar{y}_{2}^{2}=3 \bar{x}_{2}^{4}+2 .
$$

(iii) The components $\bar{Y}_{1}, \bar{Y}_{2}$ intersect in a unique split ordinary double point $\xi$ of degree 2. As a point on $\bar{Y}_{1}$, we have $\xi=(4, b)$, where $b \in \mathbb{F}_{5}(\xi)=\mathbb{F}_{5^{2}}$ is a solution to $b^{2}=3$.

(iv) The generator $\sigma$ of the Galois group $\operatorname{Gal}\left(L / \mathbb{Q}_{5}\right)=\langle\sigma\rangle$ acts trivially on $\bar{Y}_{1}$ and on $\bar{Y}_{2}$ via the automorphism of order 4

$$
\bar{x}_{2} \mapsto 3 \bar{x}_{2}, \quad \bar{y}_{2} \mapsto-\bar{y}_{2} .
$$

Proof: One simply follows the recipe in [6], $\S 4$. The equations for $\bar{Y}_{i}$, $i=1,2$, are obtained as follows. For $\bar{Y}_{1}$, we substitute $y=(x+1) y_{1}$ in (4.1), divide by $(x+1)^{4}$ and reduce modulo $\mathfrak{p}$. Using (4.2) we see that we obtain the equation 4.3. For $\bar{Y}_{2}$, we substitute $x=4+\pi x_{1}$ and $y=\pi^{2} y_{2}$, divide by 5 and reduce modulo p. A short computation yields (4.4). Statements (iii) and (iv) are straightforward.

We note that by construction the semistable model $\mathcal{Y}$ dominates the naive model $\mathcal{Y}^{\text {naive }}$, or in other words, there is a modification $\mathcal{Y} \rightarrow \mathcal{Y}^{\text {naive }} \otimes_{\mathbb{Z}_{5}} \mathcal{O}_{L}$. The resulting map $\bar{Y} \rightarrow \bar{Y}^{\text {naive }}$ may be visualized as in Figure 4.1 .
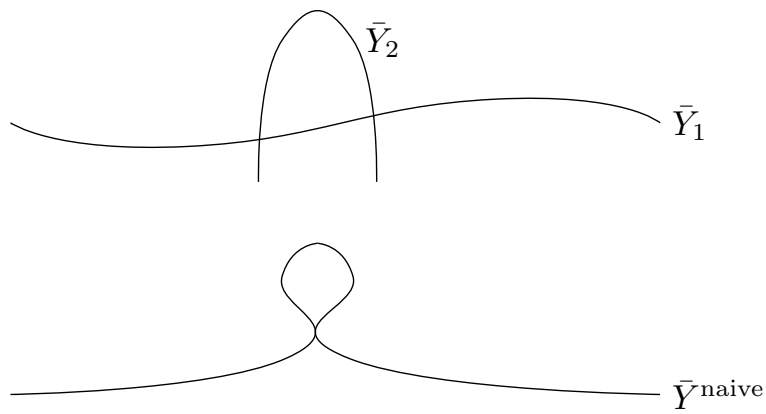

Figure 4.1: $\bar{Y} \rightarrow \bar{Y}^{\text {naive }}$

We see that $\bar{Y}_{2}$ is contracted to the singular point on $\bar{Y}^{\text {naive }}$ and that $\bar{Y}_{1}$ can be identified with the normalization of $\bar{Y}^{\text {naive }}$.

Corollary 4.2 The local $L$-factor and the conductor exponent of the curve $Y$ at $p=5$ are

$$
L_{5}^{-1}=(1+T)\left(1+3 T+5 T^{2}\right), \quad f_{5}=3 .
$$

Proof: Let $\bar{Z}:=\bar{Y} / \operatorname{Gal}\left(L / \mathbb{Q}_{5}\right)$ be the quotient of $\bar{Y}$ under the action of the Galois group of the extension $L / \mathbb{Q}_{5}$. In the terminology of $[\underline{6}, \bar{Z}$ is the inertial reduction of $Y$ at $p=5$. It follows from Lemma 4.1 that $\bar{Z}$ is a semistable curve over $\mathbb{F}_{5}$, consisting of two irreducible components $\bar{Z}_{1}, \bar{Z}_{2}$ which intersect 
in a unique non-split ordinary double point of degree 2 . The curve $\bar{Z}_{1}$ may be identified with $\bar{Y}_{1}$ and the curve $\bar{Z}_{2}$ with the quotient $\bar{Y}_{2} /\langle\sigma\rangle$. One sees immediately from (4.4) and Lemma 4.1 (iv) that $\bar{Z}_{2}$ has genus zero.

By [6], Corollary 2.5, the local $L$-factor is

$$
L_{5}(Y, s)=P\left(\bar{Z}, 5^{-s}\right)^{-1}
$$

where $P(\bar{Z}, T)$ is the numerator of the zeta function of $\bar{Z}$. From the above description of $\bar{Z}$ we see that

$$
P(\bar{Z}, T)=(1+T)\left(1+3 T+5 T^{2}\right) .
$$

The second factor is the numerator of the zeta function of the genus-one curve $\bar{Z}_{1}$, which is given by (4.3), and the first factor comes from the action of $\Gamma_{\mathbb{F}_{5}}$ on $H^{1}\left(\Delta_{\bar{Z}}\right)$, as in the proof of Proposition 2.4 Finally, we use [6], Corollary 2.6, to conclude that

$$
f_{5}=2 g_{Y}-\operatorname{dim} H_{\mathrm{et}}^{1}\left(\bar{Z}_{k}, \mathbb{Q}_{\ell}\right)=6-3=3 .
$$

We have computed the local $L$-factors and conductor exponents at all bad primes. We can now continue with Step 4 of the algorithm from $\S 3.5$. The conductor of the $L$-function is $N=3 \cdot 5^{3} \cdot 13^{2} \cdot 97 \approx 10^{8}$. We set $M:=55956$, compute the local $L$-factors for all good primes $p \leq M$ and the truncated $L$ series $L(Y, s)^{\prime}$. Feeding these data into Dokchitser's algorithm, we have checked that the $L$-function of $Y$ verifies the expected functional equation with root number -1 .

4.2 We now treat an example of a hyperelliptic curve which does not have semistable reduction at $p=2$. In this case, the methods of [6] to compute semistable reduction do not apply.

The polynomials

$$
g=x^{9}-x^{8}+x^{7}+x^{5}+x^{3}, \quad h=-x^{4}+1
$$

define a hyperelliptic curve of genus four. The discriminant of $f:=h^{2}+4 g$ is $\Delta=-2^{32} \cdot 317$. So $p=317$ is the only odd prime where $Y$ has bad reduction. Running through Step 2 and $3 \mathrm{~b}$ of the algorithm from $\S 3.5$ we see that $Y$ has semistable reduction at $p=317$ and the local $L$-factor and the conductor exponent are

$$
L_{317}^{-1}=(1+T)\left(1-32 T+991 T^{2}+\ldots\right), \quad f_{317}=1 .
$$

It will follow from the calculation of the conductor $N$ below that this is indeed the correct truncation (Remark 3.10).

Unfortunately, the condition $\operatorname{gcd}\left(\bar{h}, \bar{h}^{\prime}, \bar{g}^{\prime}\right)=1$ from $\oint$ 3.5. Step 1 , is not satisfied. The naive model $\mathcal{Y}^{\text {naive }}$ of $Y$ over $\mathbb{Z}_{(2)}$, given by (3.1), has special fiber

$$
\bar{Y}^{\text {naive }} / \mathbb{F}_{2}: \bar{y}^{2}+(\bar{x}+1)^{4} \bar{y}=\bar{g}(\bar{x})=\bar{x}^{9}+\bar{x}^{8}+\bar{x}^{7}+\bar{x}^{5}+\bar{x}^{3} .
$$


One sees at once that $\bar{Y}^{\text {naive }}$ has a non-ordinary singularity at $(\bar{x}, \bar{y})=(1,1)$. Substituting

$$
\bar{y}=(\bar{x}+1)^{3} \bar{y}_{0}+\bar{x}^{4}+\bar{x}^{2}+1
$$

into (4.5) and dividing by $(\bar{x}+1)^{6}$ we obtain an equation for the normalization $\bar{Y}_{0}^{\prime}$ of $\bar{Y}^{\text {naive }}$

$$
\bar{Y}_{0}^{\prime} / \mathbb{F}_{2}: \quad \bar{y}_{0}^{2}+(\bar{x}+1) \bar{y}_{0}=\bar{x}^{2}(\bar{x}+1) .
$$

We see that $\bar{Y}_{0}^{\prime}$ is a smooth curve of genus one. The numerator of its zeta function is

$$
P\left(\bar{Y}_{0}^{\prime}, T\right)=1+T+2 T^{2}
$$

The computation of the semistable reduction of $Y$ at $p=2$ is rather challenging. We only state the result (Lemma 4.3 below). A detailed proof will be given elsewhere.

Let us work over the field $\mathbb{Q}_{2}$ of 2-adic numbers. Using the methods of [15] we produce the following polynomial:

$$
\begin{aligned}
\Delta=x^{12} & +20 x^{11}+154 x^{10}+664 x^{9}+1873 x^{8}+3808 x^{7}+5980 x^{6} \\
& +7560 x^{5}+7799 x^{4}+6508 x^{3}+4290 x^{2}+2224 x+887 \in \mathbb{Z}_{2}[x] .
\end{aligned}
$$

One checks that $\Delta$ is irreducible over $\mathbb{Q}_{2}$. Let $L / \mathbb{Q}_{2}$ be the splitting field of $\Delta, \Gamma=\operatorname{Gal}\left(L / \mathbb{Q}_{2}\right)$ the Galois group and $K_{i}:=L^{\Gamma_{i}}$ the fixed field of the $i$ th ramification group, for $i \geq 0$. One also checks that $K_{0} / \mathbb{Q}_{2}$ has degree 2 and that $K_{1} / K_{0}$ has degree 9 . So $K_{0} / \mathbb{Q}_{2}$ is the unique unramified extension of degree 2 , and $\Gamma_{0} / \Gamma_{1}$ is a cyclic group of order 9 . Unfortunately, we do not know the exact size and structure of the wild inertia group $\Gamma_{1}$. Nevertheless, we can prove the following.

Lemma 4.3 (i) The curve $Y_{L}$ has semistable reduction.

(ii) Let $\mathcal{Y}$ be the minimal semistable model of $Y$ over $\mathcal{O}_{L}$ and $\bar{Y}$ the special fiber of $\mathcal{Y}$. Then $\bar{Y}$ consists of five irreducible components $\bar{Y}_{0}, \ldots, \bar{Y}_{4}$ over the residue field $\mathbb{F}_{\mathfrak{p}}=\mathbb{F}_{4}$ of $L$. Here $\bar{Y}_{0}$ may be identified with the pullback to $\mathbb{F}_{\mathfrak{p}}$ of the curve $\bar{Y}_{0}^{\prime}$, the normalization of $\bar{Y}^{\text {naiv }}$. The components $\bar{Y}_{1}, \bar{Y}_{2}, \bar{Y}_{3}$ are smooth curves of genus one over $\mathbb{F}_{\mathfrak{p}}$, given by equations

$$
\bar{Y}_{i} / \mathbb{F}_{p}: \bar{y}_{i}^{2}+\bar{y}_{i}=\bar{x}_{i}^{3}, \quad i=1,2,3 .
$$

The component $\bar{Y}_{4}$ is a projective line and intersects each of the other four components in a unique point. The genus one components $\bar{Y}_{0}, \ldots, \bar{Y}_{3}$ do not intersect (Figure 4.2).

(iii) The inertia group $\Gamma_{0}$ fixes $\bar{Y}_{0}$ and $\bar{Y}_{4}$ and permutes the components $\bar{Y}_{1}, \bar{Y}_{2}, \bar{Y}_{3}$ transitively. The wild inertia group $\Gamma_{1}$ fixes every component.

(iv) Let $\Gamma_{0}^{\prime} \subset \Gamma_{0}$ be the stabilizer of the component $\bar{Y}_{1}, H \subset \Gamma_{0}^{\prime}$ the kernel of the map $\Gamma_{0}^{\prime} \rightarrow \operatorname{Aut}\left(\bar{Y}_{1}\right)$, and $\tilde{\Gamma}_{0}=\Gamma_{0}^{\prime} / H$ the quotient. Then $\tilde{\Gamma}_{0}$ is cyclic of order 6 . Its unique element of order two acts on $\bar{Y}_{1}$ via the automorphism

$$
\bar{x}_{1} \mapsto \bar{x}_{1}, \quad \bar{y}_{1} \mapsto \bar{y}_{1}+1 .
$$


Moreover, the filtration of higher ramification groups on $\tilde{\Gamma}_{0}$ has the form

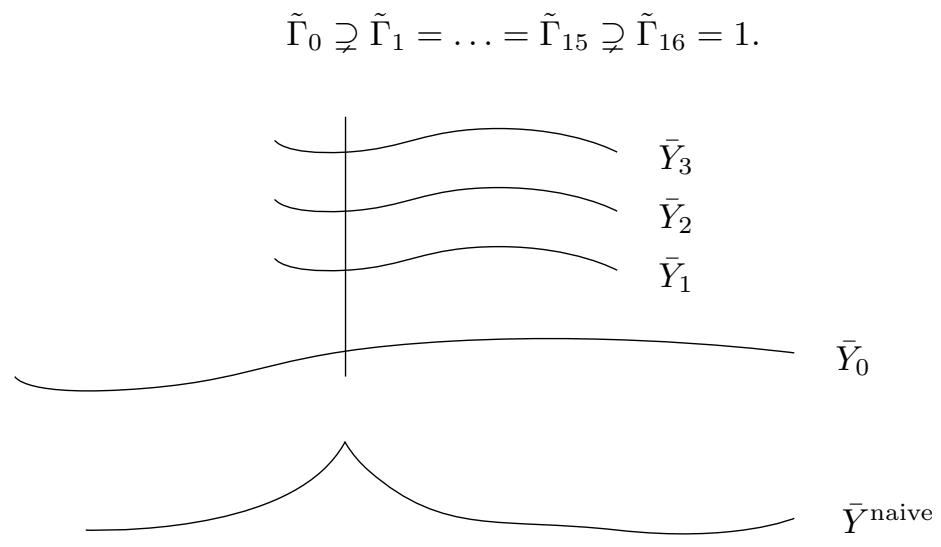

Figure $4.2: \bar{Y} \rightarrow \bar{Y}^{\text {naive }}$

Corollary 4.4 The local $L$-factor and the conductor exponent of $Y$ at $p=2$ are

$$
L_{2}^{-1}=1+T+2 T^{2}, \quad f_{2}=16 .
$$

Proof: Let $\bar{Z}=\bar{Y} / \Gamma$ be the quotient curve. It follows directly from Lemma 4.3 that $\bar{Z}$ is a semistable curve over $\mathbb{F}_{2}$, consisting of three irreducible components (corresponding to the three orbits of the action of $\Gamma_{1}$ on the set of irreducible components of $\bar{Y}$ ). The first component is the genus one curve $\bar{Z}_{0}:=\bar{Y}_{0} / \Gamma \cong \bar{Y}_{0}^{\prime}$, given by (4.6). The other two components have genus zero. Moreover, the component graph of $\bar{Z}$ is a tree. It follows that the zeta function of $\bar{Z}$ is the same as the zeta function of $\bar{Y}_{0}^{\prime}$, and hence

$$
P(\bar{Z}, T)=P\left(\bar{Y}_{0}^{\prime}, T\right)=1+T+2 T^{2},
$$

by (4.7). The claim $L_{2}^{-1}=1+T+2 T^{2}$ follows now from [6], Corollary 2.5.

By [6], $\S 2.6$, the conductor exponent $f_{2}$ has the form

$$
f_{2}=\epsilon+\delta
$$

where

$$
\epsilon=2 g_{Y}-\operatorname{dim} H_{\text {et }}^{1}\left(\bar{Z}_{k}, \mathbb{Q}_{\ell}\right)=8-2=6
$$

and $\delta=\delta_{V}$ is a $S$ wan conductor of the $\Gamma$-module $V:=H_{\mathrm{et}}^{1}\left(Y_{\overline{\mathbb{Q}}}, \mathbb{Q}_{\ell}\right)$. Since the graph of $\bar{Y}$ is a tree, the cospecialization map induces a $\Gamma$-equivariant isomorphism

$$
V \cong \oplus_{i=0}^{3} H_{\mathrm{et}}^{1}\left(\bar{Y}_{i, k}, \mathbb{Q}_{\ell}\right) .
$$

The Swan conductor of $V$ only depends on the action of $\Gamma_{0}$. By Lemma 4.3 (iii), the $\Gamma_{0}$-module $V$ has a direct sum decomposition

$$
V=V_{0} \oplus V_{1}
$$


where

$$
V_{0}=H_{\mathrm{et}}^{1}\left(\bar{Y}_{0, k}\right), \quad V_{1}=\oplus_{i=1}^{3} H_{\mathrm{et}}^{1}\left(\bar{Y}_{i, k}\right) .
$$

Moreover, $V_{0}$ has trivial $\Gamma_{0}$-action. We conclude that $\delta=\delta_{V_{1}}$ is the Swan conductor of the induced $\Gamma_{0}$-module

$$
V_{1}=\operatorname{Ind}_{\tilde{\Gamma}_{0}}^{\Gamma_{0}} \tilde{V}, \quad \tilde{V}:=H_{\mathrm{et}}^{1}\left(\bar{Y}_{1, k}, \mathbb{Q}_{\ell}\right),
$$

where the group $\tilde{\Gamma}_{0}$ is defined in Lemma 4.3 (iv). We have $\delta=\delta_{V_{1}}=\delta_{\tilde{V}}$ by standard properties of the Swan conductor. To compute $\delta_{\tilde{V}}$ we may use the formula

$$
\delta_{\tilde{V}}=\sum_{i=1}^{\infty} \frac{\left|\tilde{\Gamma}_{i}\right|}{\left|\tilde{\Gamma}_{0}\right|} \cdot \operatorname{dim} \tilde{V} / \tilde{V}^{\tilde{\Gamma}_{i}}
$$

see [6], proof of Theorem 2.9. By Lemma 4.3 (iv) we have

$$
\frac{\left|\tilde{\Gamma}_{i}\right|}{\left|\tilde{\Gamma}_{0}\right|} \cdot \operatorname{dim} \tilde{V} / \tilde{V}^{\tilde{\Gamma}_{i}}= \begin{cases}\frac{2}{6} \cdot 2, & i=0, \ldots, 15 \\ 0, & i \geq 16 .\end{cases}
$$

We conclude that $\delta=\delta_{\tilde{V}}=10$ and hence

$$
f_{2}=\epsilon+\delta=6+10=16 .
$$

It follows that the conductor of the $L$-function is $N=2^{16} \cdot 317 \approx 10^{7}$. Using the bound $M=101248$, we have verified the functional equation for $L(Y, s)$ and obtained the root number -1 .

4.3 Finally, we treat a non-hyperelliptic example. Let $Y / \mathbb{Q}$ be the superelliptic curve of genus three given by the equation

$$
y^{3}=f(x)=x^{4}-x^{2}+1 .
$$

The discriminant of $f$ is $144=2^{4} \cdot 3^{2}$. We conclude that $Y$ has good reduction at $p \neq 2,3$.

The local $L$-factor and the conductor exponent of $Y$ at $p=2$ have been computed in [6], $\S 7$. The result is

$$
L_{2}^{-1}=1+2 T^{2}, \quad f_{2}=8 .
$$

The methods of [6] do not allow the computation of the semistable reduction of $Y$ at $p=3$, because the exponent of $y$ in (4.9) is equal to $p=3$. Again, we have to use the algorithm of [15.

Let $L:=\mathbb{Q}_{3}\left[\zeta_{4}, \pi\right]$, where $\zeta_{4}$ is a primitive 4 th root of unity and $\pi$ satisfies $\pi^{12}=3$. This is a Galois extension of $\mathbb{Q}_{3}$ whose Galois group is the dihedral group of order 24 , generated by

$$
\tau\left(\pi, \zeta_{4}\right)=\left(\zeta_{12} \pi, \zeta_{4}\right) \quad \sigma\left(\pi, \zeta_{4}\right)=\left(\pi,-\zeta_{4}\right) .
$$

Here $\zeta_{12}:=\zeta_{4}^{3}\left(-1 / 2+\pi^{6} \zeta_{4} / 2\right) \in L$ is a primitive 12 th root of unity. We also put $\zeta_{3}=\zeta_{12}^{4}$. The residue field of the unique prime $\mathfrak{p}=(\pi) \triangleleft \mathcal{O}_{L}$ is $\mathbb{F}_{\mathfrak{p}}=\mathbb{F}_{3}\left[\zeta_{4}\right]=\mathbb{F}_{9}$. 
Lemma 4.5 (i) The curve $Y_{L}=Y \otimes_{\mathbb{Q}_{3}} L$ has semistable reduction at $\mathfrak{p}$.

(ii) Let $\mathcal{Y}$ be the minimal semistable model of $Y$ over $\mathcal{O}_{L}$ and $\bar{Y}$ the special fiber of $\mathcal{Y}$. Then $\bar{Y}$ consists of 4 smooth, absolutely irreducible components over $\mathbb{F}_{\mathfrak{p}}$. The normalization $\bar{Y}_{0}$ of the naive model has genus zero. The other three components $\bar{Y}_{i}(i=1,2,3)$ have genus 1 , and intersect $\bar{Y}_{0}$ in a unique ordinary double point of degree 1 (Figure 4.3). The curve $\bar{Y}$ does not have any further singularities.

(iii) The Galois group $\Gamma:=\operatorname{Gal}\left(L / \mathbb{Q}_{3}\right)$ acts trivially on $\bar{Y}_{0}$. It acts as a cyclic group $\left\langle\psi_{\tau^{3}}\right\rangle$ of order 4 on $\bar{Y}_{1}$, the quotient by this action has genus 0 .

The components $\bar{Y}_{2}$ and $\bar{Y}_{3}$ are conjugate under the action on $\bar{Y}$ induced by $\sigma$. The Galois group $\Gamma$ acts on $\bar{Y}_{2}$ (resp. $\bar{Y}_{3}$ ) as a cyclic group $\left\langle\psi_{\tau}\right\rangle$ of order 12. The quotients of $\bar{Y}_{2}$ (resp. $\bar{Y}_{3}$ ) both by $\left\langle\psi_{\tau}\right\rangle$ and by the wild subgroup $\left\langle\psi_{\tau}^{4}\right\rangle$ have genus 0 .

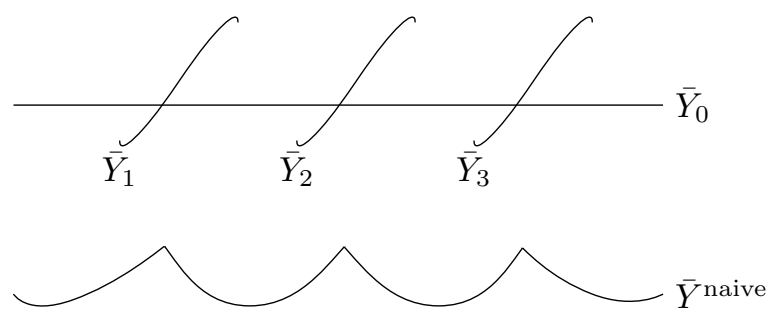

Figure 4.3: $\bar{Y} \rightarrow \bar{Y}^{\text {naive }}$

Proof: Note that $\bar{f}^{\prime}=\bar{x}\left(\bar{x}^{2}+1\right)$. It follows that the special fiber $\bar{Y}_{k}^{\text {naive }}$ of the naive model of $Y$ has singularities in the 3 points with $\bar{x}=0, \pm \zeta_{4}$. The normalization $\bar{Y}_{0}$ of $\bar{Y}_{k}^{\text {naive }}$ has genus 0 , since the map

$$
(\bar{x}, \bar{y}) \mapsto \bar{x}, \quad \bar{Y}_{k}^{\text {naive }} \rightarrow \mathbb{P}_{k}^{1}
$$

is purely inseparable.

To find the irreducible components $\bar{Y}_{i}$ for $i=1,2$ we use the coordinates

$$
\begin{aligned}
x=\pi^{9} x_{1}, & y=\left(\zeta_{3}-1\right) y_{1}+1, \\
x=a \pi^{15} x_{2}-2 \zeta_{4}, \quad y & =i \pi^{10} y_{2}+b \pi^{4}\left(-\frac{4}{7} \zeta_{4} x+1\right),
\end{aligned}
$$

where $a, b \in \mathbb{Q}_{3}[i]$ satisfy $a^{2}=\zeta_{4}$ and $b^{3}=7$. The component $\bar{Y}_{3}$ is obtained by applying the automorphism of $\bar{Y}$ induced by sending $\zeta_{4}$ to $\zeta_{4}^{3}$. Suitably normalizing $a$ and $b$, we find the following equations:

$$
\begin{array}{ll}
\bar{Y}_{1}: & \bar{y}_{1}^{3}-\bar{y}_{1}=\bar{x}_{1}^{2}, \\
\bar{Y}_{2}: & \bar{y}_{2}^{3}-\bar{y}_{2}=\bar{x}_{2}^{2} .
\end{array}
$$


Statements (i) and (ii) follow from this. Statement (iii) follows by a straightforward verification.

Corollary 4.6 The local $L$-factor and conductor exponent of $Y$ at $p=3$ are

$$
L_{3}^{-1}=1, \quad f_{3}=12 .
$$

Proof: The proof is similar to the proof of Corollaries 4.2 and 4.4 The statement on the local $L$-factor immediately follows from Lemma 4.5 (iii), since the inertial reduction $\bar{Z}$ of $Y$ at $p=3$ has genus 0 . (Here we use once more [6], Corollary 2.5.)

We compute the conductor exponent using [6], $\S 2.6$. We find that $f_{3}=\epsilon+\delta$, where

$$
\epsilon=2 g_{Y}-\operatorname{dim} H_{\mathrm{et}}^{1}\left(\bar{Z}, \mathbb{Q}_{\ell}\right)=6-0=6 .
$$

We compute the Swan conductor $\delta$ of the $\Gamma$-module $V:=H_{\text {et }}^{1}\left(\bar{Z}, \mathbb{Q}_{\ell}\right)$ using [6], Theorem 2.9. We note that the wild subgroup of the decomposition group of $\mathfrak{p}$ satisfies

$$
\tau^{4}(\pi)-\pi=\left(\zeta_{12}^{4}-1\right) \pi .
$$

Since $v_{L}\left(\zeta_{12}^{4}-1\right)=6$, we conclude that the filtration of higher ramification groups is

$$
\Gamma_{0}=\langle\tau\rangle \supsetneq \Gamma_{1}=\cdots=\Gamma_{6}=\left\langle\tau^{4}\right\rangle \supsetneq \Gamma_{7}=\{1\} .
$$

Lemma 4.5 (iii) implies that the quotient $\bar{Y} / \Gamma_{i}$ has genus one for all $1 \leq i \leq$ 6. We conclude from [6], Theorem 2.9 that

$$
\delta=\sum_{i=1}^{\infty} \frac{\left|\Gamma_{i}\right|}{\left|\Gamma_{0}\right|}\left(2 g_{Y}-2 g\left(\bar{Y} / \Gamma_{i}\right)\right)=\frac{6 \cdot 3}{12}(6-2)=6 .
$$

It follows that

$$
f_{3}=\epsilon+\delta=6+6=12 \text {. }
$$

Using the bound $M=274994$, we have verified the functional equation for $L(Y / \mathbb{Q})$ and obtained the root number 1 .

\section{References}

[1] K. Arzdorf. Semistable reduction of cyclic covers of prime power degree. $\mathrm{PhD}$ thesis, Leibniz Universität Hannover, 2012. http://edok01.tib.uni-hannover.de/edoks/e01dh12/71609648.pdf.

[2] K. Arzdorf and S. Wewers. Another proof of the semistable reduction theorem. Preprint, arXiv:1211.4624, 2012.

[3] Y. Aubry and M. Perret. Weil theorems for singular curves. In Arithmetic geometry and coding theory (Luminy, 1993), pages 1-7. de Gruyter, Berlin, 1996. 
[4] A. Booker. Numerical tests of modularity. PhD thesis, Princeton University, 2003.

[5] A. Booker. Numerical tests of modularity. J. Ramanujan Math. Soc., 20(4):283-339, 2005.

[6] I.I. Bouw and S. Wewers. Computing $L$-functions and semistable reduction of superelliptic curves. Preprint, arXiv:1211.4459,

[7] P. Deligne. Les constantes des équations fonctionelles des fonctions $L$. In Modular functions of one variable, II, number 349 in LNM, pages 501-597. Springer-Verlag, 1973.

[8] T. Dokchitser. Computing special values of motivic $L$-functions. Experimental Mathematics, 13(2):137-149, 2004.

[9] T. Dokchitser, R. de Jeu, and D. Zagier. Numerical verification of Beilison's conjecture for $K_{2}$ of hyperelliptic curves. Compositio Math., 142:339-373, 2006.

[10] P. Gaudry and R. Harley. Counting points on hyperelliptic curves over finite fields. In W. Bosma, editor, Algorithmic number theory, number 1838 in Lecture Notes in Computer Science, pages 313-332. Springer-Verlag, 2000.

[11] K.S. Kedlaya. Counting points on hyperelliptic curves using MonskyWashnitzer cohomology. Journal Ramanujan Math. Soc., 16:323-338, 2001.

[12] K.S. Kedlaya and A. Sutherland. Computing $L$-series of hyperelliptic curves. In Algorithmic Number Theory, number 5011 in Lecture Notes in Computer Science, pages 312-326. Springer-Verlag, 2008.

[13] Q. Liu. Algebraic geometry and arithmetic curves. Oxford University Press, 2006.

[14] J.S. Milne. Étale cohomology. Princeton Univ. Press, 1980.

[15] J. Rüth and S. Wewers. Semistable reduction of superelliptic curves of degree $p$. in preparation.

Michel Börner, Irene Bouw, Stefan Wewers

Institut für Reine Mathematik

Universität Ulm

Helmholtzstr. 18

$89081 \mathrm{Ulm}$

michel.boerner@uni-ulm.de, irene.bouw@uni-ulm.de, stefan.wewers@uni-ulm.de 